\title{
50 Jahre NTM
}

Die Gründung der Zeitschrift NTM vor 50 Jahren durch Gerhard Harig (1902-1966) und Alexander Mette (1897-1985) markiert einen Einschnitt in der Geschichte der deutschsprachigen Wissenschaftsgeschichte. Zu einer Zeit, in der weltweit nur an wenigen Orten ein stabiler, sich über akademische Generationen hinweg erhaltender professioneller Zusammenhang der Wissenschaftsgeschichte bestand, und zu einer Zeit, in der in beiden deutschen Staaten die Folgen des Nationalsozialismus und des Zweiten Weltkriegs das akademische Leben prägten, setzte die Gründung einer neuen, Forschungsbeiträgen gewidmeten Zeitschrift für die Geschichte der Naturwissenschaften, Technik und Medizin in der DDR ein Zeichen. Der Anspruch war hoch: Die Herausgeber wollten nicht nur die „Herkunft und [...] Geschichte der Probleme und Begriffe in Naturwissenschaft, Technik und Medizin" oder die Biographien bedeutender Wissenschaftler beleuchten, vielmehr sollte die NTM einen internationale Aufmerksamkeit und Beteiligung erreichenden Beitrag leisten zur Beantwortung der „Frage nach dem Zusammenhang zwischen der allgemeinen gesellschaftlichen Entwicklung und derjenigen der Naturwissenschaft, Technik und Medizin" (Harig/Mette 1960: 1). Die Arbeit an dieser Aufgabe sollte selbst ihren Teil zur gesellschaftlichen Entwicklung und internationalen Zusammenarbeit beitragen. Dabei wählten die Gründer ihre Worte, ohne in eine starre staatssozialistische Ideologie abzugleiten. Die NTM, so schrieben sie,

grenzt sich eindeutig und entschieden ab von allen militaristischen, faschistischen und rassistischen Richtungen und Bestrebungen und wird allen denen offenstehen, die - gleich welchem der beiden gegenwärtigen Weltlager sie angehören - für die gegenseitige Verständigung der Völker und eine friedliche Zusammenarbeit der Nationen eintreten und die Wissenschaft zum Wohle und zur Förderung des Menschengeschlechts betreiben. (Ebd.: 2 f.)

Kurz nach der Remilitarisierung der Bundesrepublik und vor dem Mauerbau hätten solche Sätze auch von manchen Autoren im Westen formuliert werden können, und obwohl die "Weltlage“ 
heute unübersichtlicher geworden ist und die politische Sprache sich verändert hat, kann man ihnen auch heute noch zustimmen.

In der frühen NTM ist das Anliegen unverkennbar, durch Beiträge zu einer breit angelegten - auch sozialgeschichtlich verstandenen - Geschichte des medizinischen, technischen und naturwissenschaftlichen Wissens die Rolle dieser Wissensfelder und der in ihnen tätigen Wissenschaftlerinnen und Wissenschaftler für den ,Fortschritt' der menschlichen Zivilisation ins Licht zu rücken.

Dass dies unter den damaligen Vorzeichen eines Staatsmarxismus geschah, und dass dieser zivilisatorische ,Fortschritt' unter Verwendung marxistischer Terminologie beschrieben wurde (etwa indem wissenschaftliche Neuerungen als Beiträge zur Entwicklung der Produktivkräfte dargestellt oder die ,humanistische' Orientierung von manchen bürgerlichen Naturwissenschaftlern und -wissenschaftlerinnen hervorgehoben wurde) ist im historischen Rückblick nicht überraschend. Vergleichbare ideologische Implikationen (nun nicht im marxistischen Sinn, sondern in jenem metahistorisch-analytischen Sinn verstanden, den Hayden White diesem Ausdruck gegeben hat) fanden sich zur gleichen Zeit unter anderen Vorzeichen auch in den wissenschaftshistorischen Agenden anderer Schauplätze. Auch im Westen war zu dieser Zeit der Glaube an den wissenschaftlichen ,Fortschritt' überraschend ungetrübt und seine Indienstnahme für das jeweilige politische System selbstverständlich. Und wo in der Wissenschaftsgeschichte der damaligen Bundesrepublik eine Kultur des Unpolitischen gepflegt wurde, mochte nicht selten auch das Schweigen der bleiernen Zeit der Nachkriegsjahre dafür verantwortlich sein.

Neben der optimistischen politischen Orientierung fällt aber am Auftakt der NTM im Jahr 1960 noch etwas anderes ins Auge, nämlich der entschiedene und selbstbewusste Wille zur Professionalisierung der Wissenschaftsgeschichte:

\footnotetext{
Entgegen gelegentlich noch anzutreffenden Ansichten ist die Geschichte der Wissenschaft heute weder eine Lieblingsbeschäftigung ergrauter Professoren noch ein einleitendes Kapitel für Lehrbücher und Monographien, sondern eine rasch wachsende, selbständige wissenschaftliche Disziplin mit eigenen Problemstellungen und Anliegen, deren Unentbehrlichkeit für die begriffliche und praktische Bewältigung der vor Wissenschaft und Gesellschaft stehenden Aufgaben immer deutlicher wird. (Ebd.: 1 f.)
}

Der Werdegang der NTM und die sich in den folgenden Jahren anschließenden Entwicklungen der Wissenschaftsgeschichte in der DDR haben gezeigt, dass diese Einschätzung nicht ganz falsch war. Im Jahr 1976 wurde ein Arbeitskreis für Wissenschaftsgeschichte 
neben dem Beirat für Geschichtswissenschaften des zuständigen Ministeriums für Hoch- und Fachschulwesen der DDR eingerichtet, der zur Aufgabe hatte, die Wissenschaftsgeschichte zu fördern. Dieses Engagement hatte zur Folge, dass die wissenschaftshistorische Lehre fester Bestandteil der Ausbildung von Oberstufenlehrern der Mathematik und Naturwissenschaften an allgemeinbildenden Schulen wurde. Im Jahr 1977 wurde vom Ministerium ein entsprechender Beschluss gefasst, der anschließend durch die gezielte Fortbildung entsprechend interessierter Dozenten der Hochschulen und den Aufbau eines einschlägigen Lehrprogramms umgesetzt wurde. Der Arbeitskreis für Wissenschaftsgeschichte wurde 1986 in einen selbstständigen Beirat umgewandelt, dessen Vorsitz Hans Wußing innehatte. Unter maßgeblicher Beteiligung von Gisela Buchheim und Rolf Sonnemann (ausführlicher berichtet hierüber Thomas Hänseroth in seinem Kommentar zum Nachdruck von Buchheims Beitrag in diesem Heft) ergaben sich zur selben Zeit ähnliche Initiativen auch im Bereich der Ingenieurwissenschaften. Die Medizingeschichte hatte aufgrund ihrer traditionellen Verankerung in der Ausbildung von Ärzten und Ärztinnen ohnehin einen festen Stand. Für alle diese Entwicklungen, die schließlich auch die Zahl der forschenden Wissenschaftshistoriker und -historikerinnen in der DDR deutlich vergrößerte, bot die NTM trotz Papiermangels und Veröffentlichungbeschränkungen ein wirksames Publikationsforum.

Für die Professionalisierung der Wissenschaftsgeschichte in der DDR war zweifellos von Bedeutung, dass führende Akteure auch in politischen Funktionen tätig waren. Gerhard Harig etwa hatte 1934 Nazideutschland verlassen und wirkte am Leningrader Institut für Geschichte der Naturwissenschaften und Technik bei der Akademie der Wissenschaften der Sowjetunion, bevor er in Folge des HitlerStalin-Pakts wieder aus der UdSSR ausgewiesen und dann in Buchenwald interniert wurde. Nach dem Ende des Krieges wurde er zunächst der erste Professor für Geschichte der Naturwissenschaften und Technik sowie für dialektischen Materialismus an der Karl-Marx-Universität Leipzig, bevor er zum Staatssekretär im Ministerium für Hoch- und Fachschulwesen berufen wurde. Von diesem Amt kehrte er allerdings schon 1957 wieder nach Leipzig zurück und erhielt die Leitung einer neuen Abteilung für Geschichte der Naturwissenschaften am dortigen Karl-Sudhoff-Institut (Mocek 1997: 99 f., Wußing/Schreier 1006: 52-55). Auch Alexander Mette war nicht nur seit 1960 Ordinarius und Direktor des Instituts für Geschichte der Medizin an der Humboldt-Universität Berlin, sondern schon seit der unmittelbaren Nachkriegszeit beim Aufbau des 
öffentlichen Gesundheitswesens in Thüringen in leitender Funktion tätig und dann ab 1950 auch Mitglied der Volkskammer.

Freilich - und auch dies zeigen die in diesem Heft versammelten Beiträge - konnten trotz (oder auch wegen) dieser Nähe zur politischen Spitze im Bereich der Geschichte der Naturwissenschaften, Technik und Medizin einige Autoren und Autorinnen der Zeitschrift eine gewisse Unabhängigkeit von der Staatsideologie durchaus wahren. In einem späteren Interview sprach Wußing von einer „elastischen Kriegführung“ mit jenen, die auch die Wissenschafts-, Technik- und Medizingeschichte der ideologisch stärker eingebundenen Theorie der Geschichtswissenschaften eingliedern wollten (Wußing 1999). Im Vergleich mit manchen anderen historischen und philosophischen Publikationen der DDR - gerade auch wo diese sich auf die Wissenschaften bezogen - kann die Agenda der Frühen NTM vielleicht tatsächlich als eine humanistische, dabei aber eben die Bedeutung der Medizin, Naturwissenschaften und Technik für die Menschheit ins Zentrum rückende Agenda bezeichnet werden. Humanismus stand hier nicht nur für eine intellektuelle Strömung der Frühen Neuzeit und auch nicht nur für ein marxistisches Konzept der Zeit des Kalten Kriegs, welches eine blockübergreifende Allianz ,fortschrittlicher' Kräfte möglich machen sollte, sondern für die aufgeklärten und liberal-demokratischen Tendenzen der gesamten Neuzeit, die man in den Wissenschaften hervorheben wollte. Dies ließ sich in vielen Einzelstudien auch dann leicht erreichen, wenn das eigentliche Forschungsanliegen eher problem- oder begriffsgeschichtlich war, also jener Richtung der Wissenschaftsgeschichte nahestand, die auch in der DDR als ,internalistisch' bezeichnet wurde. ${ }^{1}$

Insgesamt bildete die NTM damit ein Forum für eine breite, sich professionalisierende Wissenschafts-, Technik und Medizingeschichte. Der Erfolg blieb nicht aus: Zu den Autoren zählten nach und nach auch Wissenschaftshistoriker und -historikerinnen aus anderen Ländern - nicht nur aus den sozialistischen Staaten Osteuropas, sondern auch aus der Bundesrepublik, Großbritannien oder den USA.

Die Bundesrepublik hatte in den 1960er und frühen 1970er Jahren vergleichbare Erfolge der Professionalisierung der Wissenschaftsgeschichte kaum aufzuweisen. Bis in die 1980er Jahre blieben viele aktiv Forschende an ihren Universitäten eher Einzelgänger (einer der Unterzeichnenden erinnert sich an wissenschaftshistorische Vorlesungen, die es ohnehin nur an wenigen Universitäten überhaupt gab, mit sehr wenigen Hörern, drei, zwei, oder gar nur einem). Von den bedeutenden internationalen Entwicklungen der 
Wissenschaftsgeschichte hörte man damals eher in Veranstaltungen der Philosophie und Soziologie - insbesondere nachdem auch hier seit den 1970er Jahren die Rezeption der Arbeiten Thomas S. Kuhns (und dann auch rasch der Schriften von Ludwik Fleck und anderen Vertretern und Vertreterinnen der historischen Wissenschaftsforschung der Vorkriegszeit) einsetzte. Eine detaillierte Geschichte der Wissenschaftsgeschichte im deutschsprachigen Raum, welche die Entwicklungen über die politischen Brüche hinweg und für die Nachkriegszeit auch für die beiden deutschen Staaten vergleichend analysiert, ist noch nicht geschrieben. In ihr käme der NTM in jedem Fall eine interessante Rolle zu.

Nach der Vereinigung der beiden deutschen Staaten war zunächst nicht klar, wie die unterschiedlichen professionellen Strukturen der Wissenschaftsgeschichte in eine neue, gemeinsame Konfiguration übergehen konnten. Wir müssen heute feststellen, dass etliche Strukturen, die sich in der DDR entwickelt hatten, preisgegeben wurden - nicht nur eine Reihe von wissenschafts- und technikhistorischen Einrichtungen an ostdeutschen Hochschulen sowie die Arbeitsgruppe zur Wissenschaftsgeschichte an der Akademie der Wissenschaften der DDR, sondern auch die Profession tragende Strukturen wie die obligatorische Verankerung der Geschichte wissenschaftlicher und technischer Disziplinen in der Lehrerausbildung und im Studium der Ingenieurwissenschaften, ja sogar in manchem Diplomstudiengang. Dass die Zeitschrift NTM diesen Übergang mit einer neuen Redaktion und neuen Herausgebern sowie auch durch den Wechsel 1993 vom aufgelösten Teubner-Verlag zu Birkhäuser in Basel nicht nur überlebt hat, sondern im wiedervereinigten Deutschland wieder ein attraktives Journal - insbesondere auch für Nachwuchswissenschaftlerinnen und -wissenschaftler - wurde, mag als weiteres Zeichen des Erfolgs auch der ersten Periode der Zeitschrift gelten. Die vor zwei Jahren eingegangene Verbindung der Zeitschrift mit der Deutschen Gesellschaft für Geschichte der Medizin, Naturwissenschaften und Technik (die ebenfalls inzwischen einen Wandel durchlaufen hatte und zum Forum für Vertreter und Vertreterinnen der Wissenschaftsgeschichte beider früheren deutschen Staaten und verschiedener Generationen geworden war) hat der NTM noch einmal neuen Schwung gegeben, der sie weiter tragen wird.

All dies ist Grund genug, das Jubiläum eines halben Jahrhunderts zu feiern. Und was wäre da angemessener, als die Stimmen der Anfangszeit wieder zu Wort kommen zu lassen - in Verbindung mit jenen der Gegenwart. Wir drucken daher eine Auswahl von Artikeln aus den ersten dreißig Jahren hier wieder ab und fügen jeweils einen 
Kommentar aus heutiger Sicht hinzu. Beim Lesen wird rasch erkennbar, dass dabei nicht nur eine bemerkenswerte Epoche der Geschichte der deutschsprachigen Wissenschaftsgeschichte noch einmal besichtigt - und in den Kommentaren aufschlussreich analysiert - wird, sondern dass einige der theoretischen und historiographischen Impulse, die von den Beiträgen in der frühen NTM ausgingen, auch die heutige Diskussion noch anregen. Wir können darauf verzichten, diese einleitend aufzuzählen - die Texte und ihre Kommentare sprechen für sich selbst. ${ }^{2}$

Moritz Epple, Mikael Hård, Sybilla Nikolow, Hans-Jörg Rheinberger, Volker Roelcke

\section{Anmerkungen}

1 Dass es auch in der DDR eine Kontroverse zwischen ,internalistischen' und ,externalistischen' Ansätzen der Wissenschaftsgeschichte gab, bezeugt das Interview mit Hans Wußing 1999: 66.

2 Ohne Unterstützung von vielen Seiten wäre dieses Jubiläumsheft nicht zustande gekommen. Wir bedanken uns bei den Kommentatoren, die sich für dieses Vorhaben begeistern ließen, sowie bei Hans Wußing, Renate Tobies, Dieter Hoffmann, Jutta Kollesch, Hubert Laitko und Hannelore Bernhardt für die Bereitstellung der Originalbeiträge und Informationen aus erster Hand. Karin Neidhart vom Birkhäuser Verlag stand der Idee von Anfang an offen und hilfreich gegenüber. Nicht zuletzt sind wir den Autorinnen und Autoren der Beiträge sowie ihren Nachlassverwaltern zu Dank verpflichtet, die uns die Erlaubnis erteilt haben, ihre Forschungsarbeiten wieder abzudrucken.

\section{Literatur}

Harig, Gerhard/Mette, Alexander, 1960. Geleitwort. NTM. Schriftenreihe für Geschichte der Naturwissenschaften, Technik und Medizin, 1, 1-3.

Mocek, Reinhard, 1997. Naturwissenschaft und Philosophie in der DDR - ein Balanceakt zwischen Ideologie und Kognition. In: Karin Weisemann, Peter Kröner und Richard Toellner, Hg., Wissenschaft und Politik. Genetik und Humangenetik in der DDR (1949 - 1989). Münster: Lit-Verlag, 97-115.

Wußing, Hans/Schreier, Wolfgang, 2006. Zur Geschichte der Abteilung Geschichte der Naturwissenschaften am Karl-Sudhoff-Institut (1957-1966). In: Orthun Riha, Hg., 100 Jahre Karl-Sudhoff-Institut für Geschichte der Medizin und der Naturwissenschaften. Aachen: Shaker Verlag. 2006, 51-66.

Hans Wußing - Auskünfte zur Wissenschaftsgeschichte in der ehemaligen DDR. Im Gespräch mit Karl-Heinz Schlote. NTM. Internationale Zeitschrift für Geschichte und Ethik der Naturwissenschaften, Technik und Medizin, 7 (1999), 65-82. 